\title{
Cytotoxicity, and antimicrobial and physicochemical properties of sealers incorporated with Uncaria tomentosa
}

\author{
Nathália Lima CALDAS ${ }^{(a)}$ \\ Marina Carvalho PRADO(a) \\ Nancy Kudsi CARVALHO(b) \\ Plinio Mendes SENNA(c) (iD \\ Emmanuel João Nogueira Leal \\ da SILVA(a) \\ (a) Universidade do Grande Rio - Unigranrio, \\ Department of Endodontics, Rio de Janeiro, \\ RJ, Brazil \\ (b) Universidade do Estado do Rio de Janeiro \\ - UERJ, Department of EndodonticsRio de \\ Janeiro, RJ, Brazil \\ (c) Universidade do Grande Rio - Unigranrio, \\ Department of Dental Prosthesis, Rio de \\ Janeiro, RJ, Brazil
}

Declaration of Interests: The authors certify that they have no commercial or associative interest that represents a conflict of interest in connection with the manuscript.

\section{Corresponding Author:}

Emmanuel João Nogueira Leal Silva

E-mail: nogueiraemmanuel@hotmail.com

Submitted: August 23, 2020

Accepted for publication: January 21, 2021

Last revision: February 22, 2021

\begin{abstract}
This study evaluated the cytotoxicity, the antimicrobial and physicochemical properties of root canal sealers incorporated with phytotherapic Uncaria tomentosa (UT). Unmodified AH Plus (Dentsply, DeTrey, Germany) and MTA Fillapex (Angelus, Londrina, Brazil) were used as controls. UT was incorporated into AH Plus and MTA Fillapex, at concentrations of $2 \%$ and $5 \%$ of the total weight of these sealers $(w / w)$. Flowability, setting time, and solubility were evaluated following ISO requirements. The $\mathrm{pH}$ values were measured at periods of 12, 24, 48 hours, and 7 days. The antimicrobial activity of the sealers against Enterococcus faecalis was analyzed by both direct contact tests in freshly prepared sealers, and after 7 days. The cytotoxicity of the samples was evaluated by the MTT assay, to check Balb/c 3T3 cell viability. The statistical analysis was performed by one-way ANOVA and Tukey's test $(p<0.05)$. The incorporation of UT was associated with a decrease in flow, for both sealers, an increase in AH Plus setting time, increase in MTA Fillapex $\mathrm{pH}$ values, and solubility (after 14 days), for both sealers $(\mathrm{p}<0.05)$. Regarding the antibacterial evaluation, bacterial reduction was reported after incorporation of UT into both AH Plus and MTA Fillapex, up to 7 days after handling of the material $(\mathrm{P}<0.05)$. UT incorporation decreased the cytotoxic effects of both AH Plus and MTA Fillapex sealers in a way directly proportional to their respective concentrations $(p<0.05)$. In conclusion, UT can be added to both sealers to reduce their cytotoxicity, and improve their antibacterial effects, without compromising their original physicochemical properties.
\end{abstract}

Keywords: Endodontics; Phytotherapy; Root Canal Obturation; Root Canal Therapy.

\section{Introduction}

Currently, the use or incorporation of natural substances, such as medicinal plants, has gained more attention in the biomedical sciences. ${ }^{1,2}$ One of the main advantages of phytotherapy over the conventional allopathic medicines is that it causes no or hardly any adverse effects. ${ }^{1}$ Among the predilections for phytotherapic therapies, Uncaria tomentosa should be highlighted in particular. It is also known as "cat's claw," a name attributed to the small, curved spines on the 
stem at the leaf juncture. ${ }^{3}$ This herb is found in the Amazon rainforest and in other tropical areas of South and Central America. ${ }^{4}$

Uncaria tomentosa (UT) is composed of oxindole alkaloids, triterpenes, vegetal steroids, phenolic compounds, glycosides, tannin, and flavonoids. ${ }^{2,5} \mathrm{It}$ presents low toxicity and has shown antimicrobial, anti-inflammatory, antioxidant, antineoplastic and immunomodulation activity., ${ }^{2,45,6,7,8}$ UT has been widely applied in medicine, particularly in the treatment of infections, pain of diverse origin, osteoarthritis, rheumatoid arthritis, diabetes and cancer (acting as a non-specific immunomodulation agent). 2,8,9

In dentistry, this substance has shown promising results clinically in the treatment of oral candidiasis, ${ }^{10}$ and has been found to offer in vitro antimicrobial efficiency against human oral pathogens, such as Enterobacteriaceae, Streptococcus mutans and Staphylococcus spp. isolates. ${ }^{7}$ In addition, UT has been investigated as an agent for reverting problems caused by tooth bleaching procedures, owing to its antioxidant activity. ${ }^{11}$ However, the literature evaluating its action and applications is scarce in endodontics. It is known that $2 \%$ UT gel presents antimicrobial activity against the microorganisms frequently found in infected root-filled teeth. ${ }^{3}$ Furthermore, 2\% UT gel has presented antibacterial effects and substantivity similar to those of $2 \%$ chlorhexidine gel in root dentin contaminated with Enterococcus faecalis, when used as an irrigant during chemo-mechanical preparation. ${ }^{12}$

The goal of endodontic therapy is to eliminate microorganisms from the root canal system, mainly by means of chemo-mechanical preparation. Additionally, it also pursues the perpetuation of this disinfection by filling the intraradicular space with biocompatible materials. Since the complete elimination of microorganisms is not considered feasible in the present day and age, widespread interest has focused on developing filling materials with improved antimicrobial activity. ${ }^{13,14}$ Consequently, the incorporation of filling materials with UT - a substance with known antimicrobial action against endodontic pathogens - seems beneficial and promising. Another consideration is the low toxicity and anti-inflammatory action of UT. Root canal sealers may come in contact with periradicular tissues during endodontic therapy; however, the commonly used materials present varying degrees of toxic effects. ${ }^{15,16}$ The incorporation of UT into endodontic sealers may reduce such cytotoxic properties, and thus provide satisfactory outcomes. Nevertheless, the properties of endodontic materials after incorporation of UT have never been compared before in the literature. Therefore, the aim of the present study was to evaluate the cytotoxicity, and the antimicrobial and physicochemical properties of root canal sealers after the incorporation of phytotherapic Uncaria tomentosa. The null hypothesis tested was that the incorporation of UT does not interfere in these properties of root canal sealers.

\section{Methodology}

\section{Experimental groups, preparation and incorporation of Uncaria tomentosa}

AH Plus (Dentsply, DeTrey, Konstanz, Germany) and MTA Fillapex (Angelus, Londrina, Brazil) sealers were prepared according to the instructions provided by their respective manufacturers, and used as controls in an unmodified composition.

Uncaria tomentosa was obtained commercially for use in this study (powder - 100\% purity; Doce Flora, Duque de Caxias, Brazil). Handling of the material included incorporation of UT into these sealers, in concentrations of $2 \%$ or $5 \%$ of the total weight, yielding a total of 6 groups to be evaluated in this study: AH Plus (Control - 0\% UT), AH Plus $2 \%$ (AH Plus + 2\% UT), AH Plus 5\% (AH Plus + 5\% UT), MTA Fillapex (Control - 0\% UT), MTA Fillapex $2 \%$ (MTA Fillapex $+2 \%$ UT), and MTA Fillapex 5\% (MTA Fillapex + 5\% UT).

\section{Flow evaluation}

The flow test was performed following the standards set by the International Standards Organization (ISO) 6876:2012. After the material was handled, a total of three $0.05-\mathrm{mL}$ samples of each of the 6 groups evaluated was delivered onto the center of a glass plate (height/width: 40 $\mathrm{mm} / 40 \mathrm{~mm}$; thickness: $5 \mathrm{~mm}$ ). After 180 seconds from initiating the mixing, a second glass plate with the same dimensions, and weighing $20 \mathrm{~g}$, was 
placed centrally on top of the material, followed by a 100-g weight. Therefore, a total weight of 120 $\mathrm{g}$ was applied. Ten minutes after the mixing was initiated, the total weight was removed and three measurements were taken of each material. The measurements were determined by obtaining the mean of the maximum and minimum diameters of compressed disk from the sealers, using a digital caliper (Mitutoyo MTI Corporation, Tokyo, Japan). The flow test was repeated when two diameters differed more than $1 \mathrm{~mm}$ for any sealer. The mean values and standard deviation $(\mathrm{mm})$ of each sealer were calculated and recorded to obtain the flow rates. The mean of three measurements for each group was taken to represent the flow of the material.

\section{Setting time evaluation}

The setting time of the control and the experimental sealers was determined according to ISO 6876:2012. A total of 3 samples of each sealer were handled and inserted into stainless steel ring molds (internal diameter: $10 \mathrm{~mm}$; thickness: $2 \mathrm{~mm}$ ), which were placed on a flat glass plate (thickness: $1 \mathrm{~mm}$ ). After $120 \pm 10 \mathrm{~s}$ from handling, the samples were stored at $37^{\circ} \mathrm{C}$ with $95 \%$ relative humidity. After $150 \pm 10$ s from initiating the mixing, a Gilmore needle weighing $100 \pm 0.5 \mathrm{~g}$ and measuring $2.1 \mathrm{~mm}$ in diameter was placed on the material surface. This procedure was repeated until no indentations could be detected on the surface of the sealers, and the time was recorded as the setting time. Mean values and standard deviation (min) of each sealer were then calculated.

\section{Solubility evaluation}

The solubility test was performed according to ISO 6876:2012, with modifications. ${ }^{17}$ Three samples of each sealer were placed into polyvinyl chloride (PVC) molds (internal diameter: $8 \mathrm{~mm}$; thickness: $1.6 \mathrm{~mm})$, and weighed using a precision analytical balance (0.001-g accuracy; Chyo JK-180, Chyo Balance, Japan). The mean value obtained for each material was recorded as the initial weight. Then, the samples were left to set for $24 \mathrm{~h}$ on a grating table in an incubator at $37^{\circ} \mathrm{C}$ and $100 \%$ relative humidity. Afterwards, each sample was placed in a cylindrical polystyrene-sealed container with $20 \mathrm{~mL}$ of deionized water at $37^{\circ} \mathrm{C}$. PVC matrices were used as a support for each sample, and prevented any contact to be made between the surface of the material and the container, thereby maintaining the surface of the sealers in complete contact with water.

After 7 and 14 days, the samples were removed from this apparatus, blotted dry to constant weight (dry weight) at $37^{\circ} \mathrm{C}$ for $48 \mathrm{~h}$, and finally discarded. If a specimen showed any sign of disintegration, it was discarded and the test was repeated. The solubility value for each material, represented by the percentage of weight variation, was measured at each time period according to the following formula:

Solubility $=\left(\frac{\text { Dry weight at time } t-\text { Initial weight }}{\text { Initial weight }}\right) \times 100$

\section{pH evaluation}

The $\mathrm{pH}$ was tested by inserting the sealers into polyethylene tubes measuring $1.0 \mathrm{~mm}$ in internal diameter and $10.0 \mathrm{~mm}$ in length, with one closed end, using a Lentulo spiral (Dentsply Maillefer, Ballaigues, Switzerland). Five samples were used for each group. Standardization was confirmed by weighing the amount of each material in each tube $( \pm 0.002 \mathrm{~g})$. Then, the specimens were immediately immersed in glass test tubes with $10 \mathrm{~mL}$ of deionized water. The $\mathrm{pH}$ was checked prior to immersion of the specimens, to avoid any interference in the results. Next, the tubes were sealed with Parafilm (American National Can, Menasha, USA) and stored at $37^{\circ} \mathrm{C}$ during a period of $12 \mathrm{~h}, 24 \mathrm{~h}, 48 \mathrm{~h}$ or 7 days. The samples were removed at these respective time periods, the tubes were agitated in a vortex for $5 \mathrm{~s}$, and the soaking water was collected and renewed. Then, the $\mathrm{pH}$ values of the liquid obtained were recorded using a $\mathrm{pH}$ meter (266S; Orion, USA) previously calibrated with solutions of known $\mathrm{pH} .{ }^{15}$

\section{Antimicrobial activity}

The direct contact test was applied to measure the antimicrobial activity of the sealers. ${ }^{18}$ Pure cultures of Enterococcus faecalis strains (ATCC 29212) were used in the present study. The strains were grown overnight at $37^{\circ} \mathrm{C}$ in trypticase soy broth 
(Difco, Detroit, USA) supplemented with $1 \%$ glucose (Merck, Whitehouse Station, USA). After confirming the bacterial growth in a solid medium, E. faecalis strains were suspended in a tube containing $5 \mathrm{~mL}$ of brain heart infusion (BHI, Lab M, Bury, UK). The bacterial inoculum was standardized to an optical density of $0.1(600 \mathrm{~nm})$.

A sterile 96-well polystyrene microtiter plate (3599 Costar Corning model; NY, USA) was filled with exactly $30 \mathrm{~mL}$ of each sealer, in a pre-established area on the side wall of the wells, using a cavity liner applicator. The materials were analyzed at two evaluation periods: freshly prepared (after 20 min of handling) and after 7 days, in a humid atmosphere at $37^{\circ} \mathrm{C}$. Then, a $10 \mathrm{~mL}$ aliquot of bacterial suspension (with approximately $3 \times 10^{6}$ bacteria) was administered with caution on the outer face of the sealers. In the positive control group, the bacterial suspension was applied to the walls of the uncoated wells, whereas, in the negative control group, no bacterial suspension was applied to the walls of the sealer-coated wells.

After incubation of the wells in $100 \%$ humidity, at $37^{\circ} \mathrm{C}$ for $60 \mathrm{~min}, 240 \mathrm{~mL}$ of tryptic soy agar (TSB; Becton, Spark, USA) was added to each of the 96-well plates. Then, the bacterial suspension from each well was gently stirred with a sterile pipette, for $1 \mathrm{~min}$, transferred to microtiter plates and subjected to 10-fold serial dilutions in sterile saline. The bacteria survival rate was assessed by culturing $20-\mathrm{mL}$ aliquots of each dilution performed on TSA plates. Colony-forming units were counted after incubation of the plates for $24 \mathrm{~h}$ at $37^{\circ} \mathrm{C}$. All group experiments were performed in triplicate.

\section{Cytotoxicity}

The sealers were mixed according to the instructions provided by the manufacturer, and placed in sterile cylindrical Teflon rings (diameter: 5 $\mathrm{mm}$; height: $2 \mathrm{~mm}$ ) under aseptic conditions. When excess flash material was observed, it was removed with a sterile scalpel. After $24 \mathrm{~h}$, the sealers were removed from the Teflon rings and placed in Dulbecco modified Eagle medium (DMEM) (Gibco, Grand Island, USA), supplemented with $10 \%$ fetal bovine serum (FBS) (Sigma-Aldrich, St. Louis, USA), 100 $\mathrm{mg} / \mathrm{mL}$ streptomycin, and $100 \mathrm{mg} / \mathrm{mL}$ penicillin, in a surface area to volume ratio of approximately $150 \mathrm{~mm}^{2} / \mathrm{mL}$ between the surface of the samples and the volume of the medium..$^{15,19}$ The extraction vials were agitated for $24 \mathrm{~h}$ in a water bath, at $37^{\circ} \mathrm{C}$. The control samples containing only culture medium were treated as previously explained. Undiluted (1:1), 1:2, 1:4 and 1:8 extracts were used to do the testing. Balb/c 3T3 cells (American Tissue Type Collection, Manassas, USA) were cultured in DMEM (Gibco, Grand Island, USA) supplemented with 10\% FBS (Sigma-Aldrich, St. Louis, USA), $100 \mathrm{mg} / \mathrm{mL}$ streptomycin, and $100 \mathrm{mg} / \mathrm{mL}$ penicillin at $37^{\circ} \mathrm{C}$ in a humidified incubator under ambient atmospheric air pressure, and containing $5 \% \mathrm{CO}_{2}$. Confluent cells were detached with $0.25 \%$ trypsin and $0.05 \%$ EDTA for $5 \mathrm{~min}$, and aliquots of separated cells were subcultured. The experimental setup consisted of seeding cells in 24 well plates $\left(1 \times 10^{5}\right.$ cells/well), and allowing them to achieve $80 \%$ confluence (overnight attachment). Afterwards, the culture medium was replaced with $500 \mu \mathrm{L}$ of the medium eluted from the materials, for a period of 24 hours. The control group consisted of cells exposed to $500 \mu \mathrm{L}$ of cell culture medium without sealer elution for 24 hours.

Cell viability was determined using the 3-(4,5-dimethylthiazol-2-yl)-2,5-diphenyl tetrazolium bromide (MTT) assay. The culture medium was removed from each well, and the cells were washed gently with $1 \mathrm{~mL}$ phosphate-buffered saline. The wash was replaced with an MTT-succinate solution (1 mg/mL; Sigma- Aldrich, St Louis, USA), and remained incubated for $4 \mathrm{~h}$. Then, the solution was aspirated, the cell monolayers were rinsed with double-distilled water, and the water was completely removed. Formazan crystals were produced within the cells using a succinate dehydrogenase reduction of MTT, and were dissolved using distaining solution (isopropanol-10\%NP40-0.4N HCl). Then, $100-\mu \mathrm{L}$ aliquots of the solution were transferred from each well to a 96-well plate, and the absorbance was measured at $490 \mathrm{~nm}$, using a microplate reader (Urit 660, Urit, Guillin Guanxi, China). The formazan content of each well was computed as a percentage of the control group (untreated cells). Three replicates of each experiment were performed. 


\section{Statistical analysis}

The data for each test performed were statistically tabulated and analyzed for normality using the Kolmogorov-Smirnov test. Afterwards, the data were analyzed using one-way ANOVA, and the statistical differences among the groups were analyzed using the Tukey test, with a significance level set at $5 \%$.

\section{Results}

\section{Physicochemical properties}

Overall results showed that the incorporation of UT interferes in the physicochemical properties of AH Plus and MTA Fillapex (Table). When comparing the original formulation of the sealers, AH Plus had lower flowability, $\mathrm{pH}$ and solubility than MTA Fillapex $(\mathrm{p}<0.05)$. As regards AH Plus and MTA Fillapex sealers incorporated with UT, the setting time and the flowability were influenced significantly by the addition of this phytotherapic. Higher concentrations of UT were associated with longer periods of setting time for AH Plus, and lower flowability of both sealers $(p<0.05)$. Therefore, the low concentration of UT ( $2 \%)$ performed better among the UT-incorporated AH Plus sealers. MTA Fillapex did not set completely in neither its original nor its UT-containing formulation, even after 7 days had elapsed.

AH Plus showed a neutral $\mathrm{pH}$, and no difference among the analysis periods, with or without the addition of UT ( $p>0.05)$. In contrast, the MTA Fillapex groups maintained an alkaline $\mathrm{pH}(\sim 9.0)$ in all experimental periods, but smaller $\mathrm{pH}$ values after $48 \mathrm{~h}$ $(\sim 8.0)$ and 7 days $(\sim 7.7)(\mathrm{p}<0.05)$. The incorporation of $5 \%$ UT in the composition of MTA Fillapex increased its $\mathrm{pH}$ values $(\mathrm{p}<0.05)$, thus offering better results than the incorporation of $2 \%$ UT. Solubility was not affected at 7 days ( $p>0.05$ ), but increased in the UT groups after 14 days $(p<0.05)$.

\section{Antimicrobial activity}

The antimicrobial activity of AH Plus was similar to that of MTA Fillapex in freshly mixed conditions ( $p>0.05)$; however, MTA Fillapex showed higher antimicrobial activity in the set samples $(p<0.05)$ (Figure 1). Moreover, when sealers were freshly tested, they presented higher antibacterial activity than after 7 days of setting $(p<0.05)$. The incorporation of UT decreased the bacterial values presented by the control sealers in set specimens $(\mathrm{p}<0.05)$ (Figure 1).

\section{Cytotoxicity}

MTA Fillapex groups demonstrated higher cytotoxic values in comparison with the AH Plus groups $(p<0.05)$ (Figure 2). The incorporation of UT decreased the cytotoxic effects of the sealers proportionally to its concentration $(p<0.05)$.

Table. Results for the physicochemical properties (mean \pm standard deviation) of the sealers evaluated: wt\%, percentage weight variation.

\begin{tabular}{|c|c|c|c|c|c|c|}
\hline \multirow{3}{*}{ Property } & \multicolumn{3}{|c|}{ AH Plus } & \multicolumn{3}{|c|}{ MTA Fillapex } \\
\hline & \multirow{2}{*}{ AH Plus } & AH Plus & AH Plus & \multirow{2}{*}{ MTA Fillapex } & MTA Fillapex & MTA Fillapex \\
\hline & & $2 \%$ & $5 \%$ & & $2 \%$ & $5 \%$ \\
\hline Setting time (min) & $527 \pm 15^{A}$ & $588 \pm 15^{B}$ & $614 \pm 9 c$ & - & - & - \\
\hline Flow (mm) & $21.3 \pm 0.9^{A}$ & $19.1 \pm 0.3^{B}$ & $15.3 \pm 0.4^{c}$ & $30.7 \pm 0.3^{A^{*}}$ & $28.6 \pm 0.4^{\mathrm{B}^{*}}$ & $25.1 \pm 0.6^{c^{*}}$ \\
\hline $\mathrm{pH}-12$ hours & $7.1 \pm 0.1^{\mathrm{Aa}}$ & $7.2 \pm 0.1^{\mathrm{Aa}}$ & $7.3 \pm 0.2^{\mathrm{Aa}}$ & $8.8 \pm 0.3^{\mathrm{Aa}^{*}}$ & $9.0 \pm 0.1^{\mathrm{ABa}^{*}}$ & $9.3 \pm 0.1^{\mathrm{Ba}}$ \\
\hline $\mathrm{pH}-24$ hours & $7.1 \pm 0.1^{\mathrm{Aa}}$ & $7.1 \pm 0.1^{\mathrm{Aa}}$ & $7.2 \pm 0.2^{\mathrm{Aa}}$ & $8.5 \pm 0.3^{\mathrm{Aa}^{*}}$ & $8.7 \pm 0.1^{\mathrm{Aa}^{*}}$ & $9.2 \pm 0.1^{\mathrm{Ba}^{*}}$ \\
\hline $\mathrm{pH}-48$ hours & $6.8 \pm 0.1^{\mathrm{Aa}}$ & $6.9 \pm 0.2^{\mathrm{Aa}}$ & $6.9 \pm 0.2^{\mathrm{Aa}}$ & $7.8 \pm 0.1^{\mathrm{Ab} *}$ & $8.2 \pm 0.1^{A b^{*}}$ & $8.1 \pm 0.1^{A b^{*}}$ \\
\hline $\mathrm{pH}-7$ days & $6.9 \pm 0.1^{\mathrm{Aa}}$ & $6.9 \pm 0.1^{\mathrm{Aa}}$ & $7.0 \pm 0.1^{\mathrm{Aa}}$ & $7.4 \pm 0.1^{\mathrm{Ab}}$ & $7.7 \pm 0.1^{\mathrm{ABb}^{*}}$ & $7.9 \pm 0.1^{\mathrm{Bb}}$ \\
\hline Solubility (wt\%) - 7 days & $0.18 \pm 0.1^{\mathrm{Aa}}$ & $0.19 \pm 0.1^{\mathrm{Aa}}$ & $0.23 \pm 0.1^{\mathrm{Aa}}$ & $7.12 \pm 0.5^{\mathrm{Aa}^{*}}$ & $7.39 \pm 3.6^{\mathrm{Aa}^{*}}$ & $7.71 \pm 1.7^{\mathrm{Aa}^{*}}$ \\
\hline Solubility (wt\%) - 14 days & $0.38 \pm 0.1^{\mathrm{Ab}}$ & $0.58 \pm 0.3^{\mathrm{Bb}}$ & $0.61 \pm 0.1^{\mathrm{Bb}}$ & $14.2 \pm 2.2^{\mathrm{Ab}^{*}}$ & $16.9 \pm 3.8^{8 b^{*}}$ & $17.8 \pm 1.3^{\mathrm{Bb}}$ \\
\hline
\end{tabular}

*Indicate significant differences between AH Plus and MTA Fillapex at the same concentrations of Uncaria Tomentosa. Uppercase superscript letters ${ }^{A-C}$ indicate statistically different values $(p<0.05)$ between the different Uncaria Tomentosa concentrations in the same sealer. Lowercase superscript letters ${ }^{a-d}$ indicate statistically different values for flow and solubility tests $(p<0.05)$ among the different time periods (hours/days). 


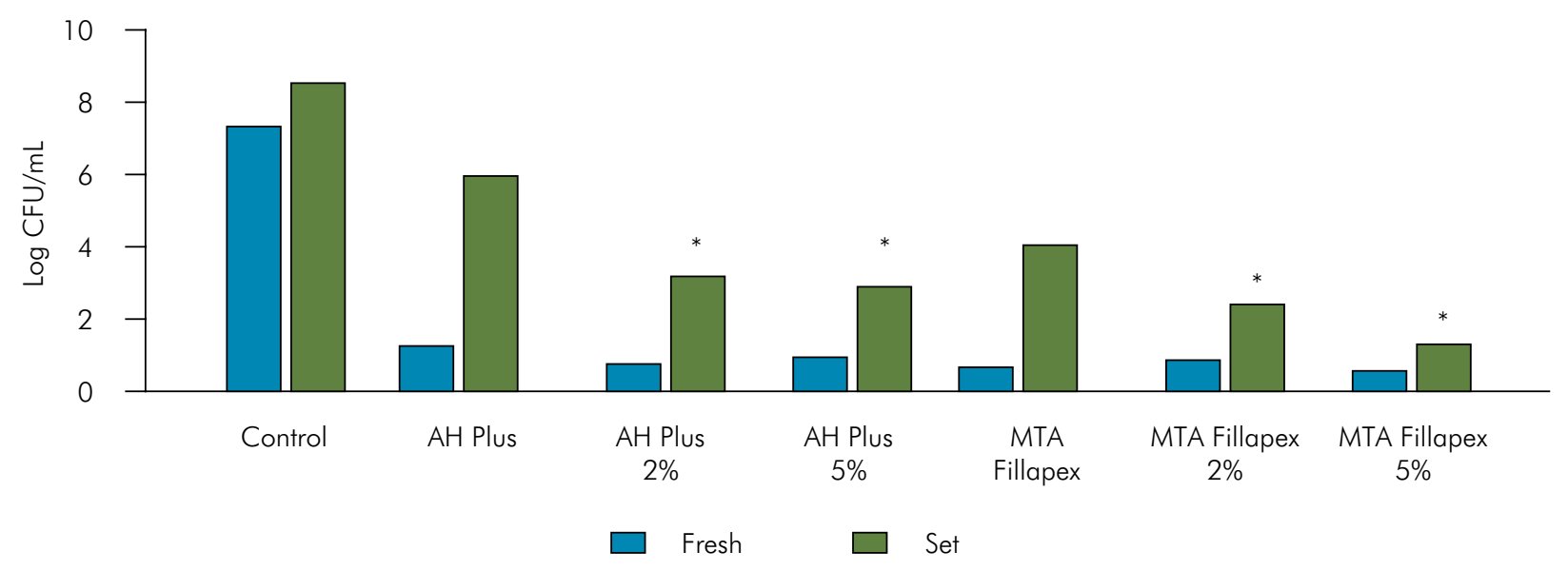

*Value differs significantly from the unmodified sealers with no Uncaria Tomentosa incorporation at the same time period $(p<0.05)$.

Figure 1. Antimicrobial activity (CFU/mL) of tested sealers both freshly prepared and after 7 days of handling.

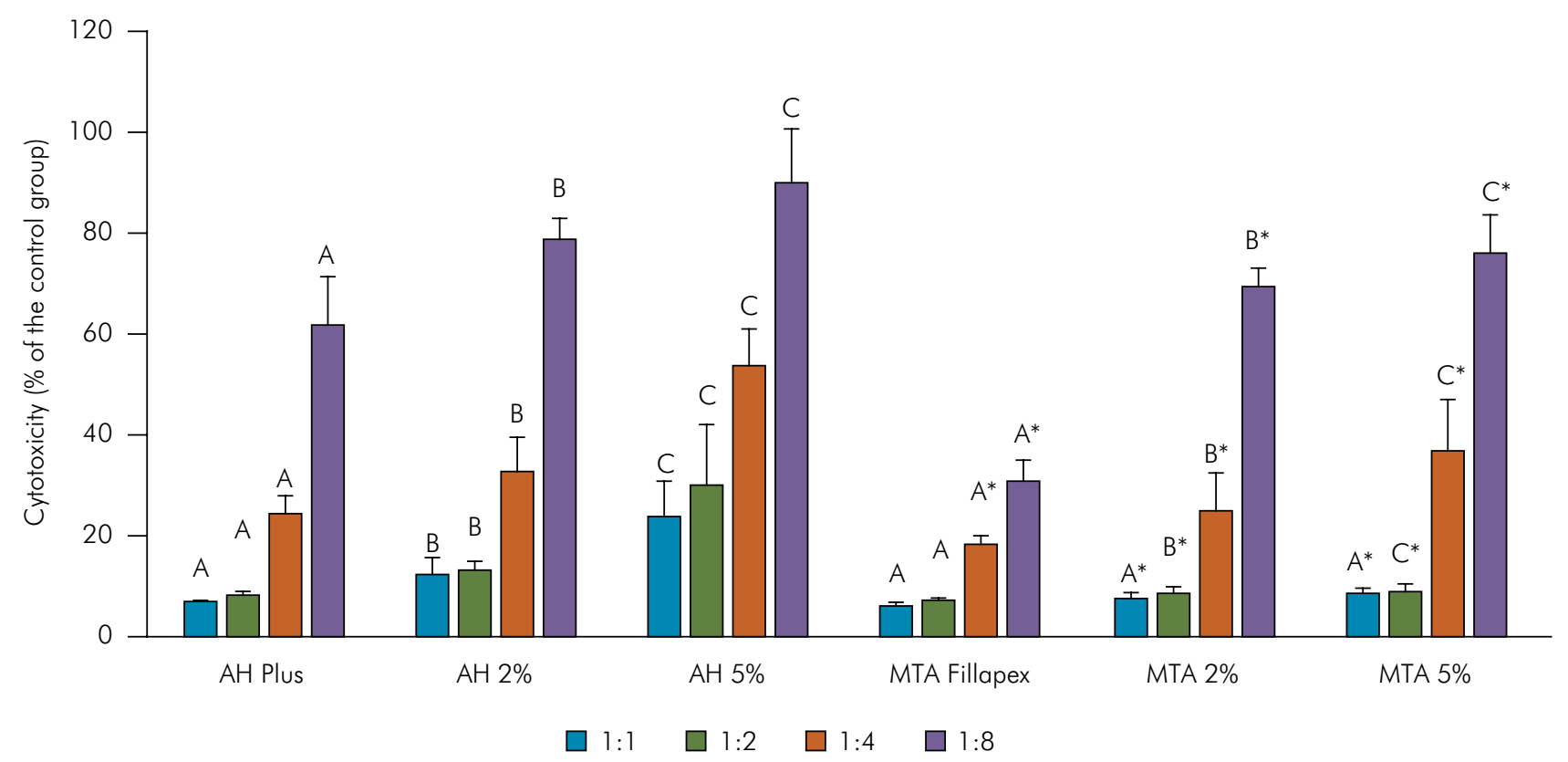

*Represents statistical differences between AH Plus and MTA Fillapex in corresponding Uncaria Tomentosa concentrations, within the same material dilution $(1: 1,1: 2,1: 4$ and $1: 8)$. Different uppercase letters indicate a statistically significant difference $(p<0.05)$ among the different UT concentrations of the same sealer, within the same material dilution $(1: 1,1: 2,1: 4$ and 1:8).

Figure 2. Cytotoxic results after exposure of fibroblast cells to tested sealers. Results are expressed as mean and standard deviation at different dilution percentages.

\section{Discussion}

Previous studies have shown the antimicrobial efficiency of Uncaria tomentosa against microorganisms commonly detected in the root canal system, ${ }^{3,12}$ and its anti-inflammatory action, ${ }^{5,8}$ factors that encourage investigations into its use for root canal treatment. In this study, the incorporation of UT was found to have influenced the antimicrobial, biological and physicochemical results of AH Plus and MTA Fillapex sealers. Therefore, the null hypothesis tested was 
rejected. The use of experimental groups with $2 \%$ UT concentration was based on previous studies., ${ }^{3,12}$ A $2 \%$ UT gel formulation is known to have antimicrobial activity against the microorganisms frequently found in infected root-filled teeth. ${ }^{3}$ In addition, $2 \%$ UT gel showed antibacterial effects and substantivity similar to those of $2 \%$ chlorhexidine gel in root dentin contaminated with Enterococcus faecalis..$^{12}$ The reason for selecting a maximum concentration of $5 \%$ is because higher concentrations tend to interfere more significantly in material properties, and also because pilot studies have shown that UT concentrations higher than $5 \%$ have had a negative influence on the physicochemical properties of sealers.

The incorporation of UT at final concentrations of $2 \%$ and $5 \%$ was significantly and proportionally associated with an increase in the antibacterial action of the set root canal sealers. This finding corroborates that of previous literature, in which UT was found to have antiviral and antibacterial activity. ${ }^{3,6,7,12}$ The UT contain some active compounds that have been previously associated with an antimicrobial effect, including dichloromethane and methanol extracts. ${ }^{2,6}$ Moreover, isopteropodine has shown antibacterial activity against Gram-positive bacteria, and is considered the strongest antimicrobial component of the UT species. ${ }^{6}$ Further studies are needed to evaluate the correlation between the chemical compounds that compose UT and antimicrobial activity, in addition to its specific mechanism of action.

One of the main features of endodontic materials is cytotoxicity, since they may extrude to periapical regions during conventional filling techniques. Therefore, these frequently used materials cause toxic effects. ${ }^{15,16} \mathrm{AH}$ Plus is considered a gold-standard root canal sealer that presents moderate cytotoxicity when freshly prepared, but becomes less or non-cytotoxic after setting. ${ }^{15,16,20} \mathrm{AH}$ Plus is an epoxy resin-based sealer, whose cytotoxicity is associated with the release of formaldehyde in association with amine and epoxy resin components..$^{14,15}$ MTA Fillapex is another widely used sealer, but is reported to have substantial and long-lasting cytotoxic effects, attributed to high levels of toxic resinous components. ${ }^{15,20}$ In the present study, the incorporation of UT extracts may have interfered in the final reaction products of all AH Plus and
MTA Fillapex groups, thus reducing cytotoxic values. Furthermore, although a positive correlation has been observed in the literature between the antimicrobial and the cytotoxicity effects of resinous sealers with antibacterial additives (e.g., natural extracts, quaternary ammonium compounds, and chlorhexidine), ${ }^{14}$ the incorporation of UT both increased antibacterial action and decreased the cytotoxic values of the sealers proportionally to its concentration.

Another relevant issue in regard to the contact made by endodontic sealers with periradicular tissues is the anti-inflammatory action of UT. This property has been previously associated with the presence of quinovic acid (glycoside 7, with a C-3, -27 glycosylation pattern). ${ }^{5}$ However, one of the major bioactive antiinflammatory components of UT is mitraphylline, which is capable of modulating cytokines, thus promoting a decrease in inflammatory signaling. ${ }^{2,8}$ Consequently, this anti-inflammatory property of UT, together with its effect in decreasing both the cytotoxicity of sealers and bacterial formation, makes its incorporation highly beneficial in $\mathrm{AH}$ Plus and MTA Fillapex sealers. The incorporation of UT may display anti-bacterial action in a filled root canal, and may also have the potential to reduce the harmful effects of bacteria in periradicular tissues during extrusion, including neurotoxic damage and inflammation.

It is important to emphasize that the incorporation of antibacterial substances into root canal sealers should be undertaken only if it does not deteriorate the physicochemical properties of the materials. The incorporation of UT interfered significantly in the physicochemical properties of AH Plus and MTA Fillapex. UT was associated with the lower flowability and higher solubility of both sealers after 14 days, as well as longer setting periods for AH Plus, and increased $\mathrm{pH}$ values for MTA Fillapex. Even though the incorporation of UT altered the results, the physicochemical properties of UT sealers remained acceptable and within respective ISO requirements. The only exception regards the flow rate of AH Plus $5 \%$. Furthermore, the setting time and solubility of all MTA Fillapex groups were considered inadequate regarding each variable. In its original formulation, MTA Fillapex did not set completely, even after 7 days 
had elapsed, and presented solubility rates that were twice as high as the maximum recommended value. These are matters of concern, since they predispose the material to leakage, and also interfere in its dimensional stability, factors that may lead to undesirable outcomes in endodontic therapy. ${ }^{14,21}$

Phytotherapic UT was shown to increase antibacterial activity, while decreasing the cytotoxic effects of the root canal sealers commonly used in root canal treatment. These results are of clinical relevance, since they reveal important features for securing promising endodontic outcomes without impairing the physicochemical properties of root canal sealers. The present findings encourage future studies to investigate the incorporation of this phytotherapy in root canal sealers, assess other properties, and determine if the present results for AH Plus and MTA Fillapex are replicable in other types of endodontic sealers.

\section{Conclusions}

In conclusion, the incorporation of phytotherapic Uncaria tomentosa decreased cytotoxicity, and raised the antimicrobial action of root canal sealers, without compromising their original physicochemical properties. The recommended concentrations of Uncaria tomentosa are 2\% and 5\% for AH Plus and MTA Fillapex, respectively, since these concentrations presented improved cytotoxicity and antimicrobial effects, and were still within the ISO requirements for physicochemical properties.

\section{References}

1. Colalto C. What phytotherapy needs: evidence-based guidelines for better clinical practice. Phytother Res. 2018 Mar;32(3):413-25. https://doi.org/10.1002/ptr.5977

2. Serrano A, Ros G, Nieto G. Bioactive compounds and extracts from traditional herbs and their potential anti-inflammatory health effects. Medicines (Basel). 2018 Jul;5(3):E76. https://doi.org/10.3390/medicines5030076

3. Herrera DR, Tay LY, Rezende EC, Kozlowski VA Jr, Santos EB. In vitro antimicrobial activity of phytotherapic Uncaria tomentosa against endodontic pathogens. J Oral Sci. 2010 Sep;52(3):473-6. https://doi.org/10.2334/josnusd.52.473

4. De Martino L, Martinot JL, Franceschelli S, Leone A, Pizza C, De Feo V. Proapoptotic effect of Uncaria tomentosa extracts. J Ethnopharmacol. 2006 Aug;107(1):91-4. https://doi.org/10.1016/i.jep.2006.02.013

5. Aquino R, De Feo V, De Simone F, Pizza C, Cirino G. Plant metabolites. New compounds and anti-inflammatory activity of Uncaria tomentosa. J Nat Prod. 1991 Mar-Apr;54(2):453-9. https://doi.org/10.1021/np50074a016

6. García R, Cayunao C, Bocic R, Backhouse N, Delporte C, Zaldivar M, et al. Antimicrobial activity of isopteropodine. Z Natforsch C J Biosci. 2005 May-Jun;60(5-6):385-8. https://doi.org/10.1515/znc-2005-5-603

7. Ccahuana-Vasquez RA, Santos SS, Koga-Ito CY, Jorge AO. Antimicrobial activity of Uncaria tomentosa against oral human pathogens. Braz Oral Res. 2007 Jan-Mar;21(1):46-50. https://doi.org/10.1590/S1806-83242007000100008

8. Rojas-Duran R, González-Aspajo G, Ruiz-Martel C, Bourdy G, Doroteo-Ortega VH, Alban-Castillo J, et al. Antiinflammatory activity of Mitraphylline isolated from Uncaria tomentosa bark. J Ethnopharmacol. 2012 Oct;143(3):801-4. https://doi.org/10.1016/i.jep.2012.07.015

9. Della Valle V. Uncaria tomentosa. G Ital Dermatol Venereol. 2017 Dec;152(6):651-7.

10. Paiva LC, Ribeiro RA, Pereira JV, Oliveira NM. Avaliação clínica e laboratorial do gel da Uncaria tomentosa (Unha de Gato) sobre candidose oral. Rev Bras Farmacol. 2009 Apr/Jun;19(2a 2A):423-8. https://doi.org/10.1590/S0102-695X2009000300015

11. Garcia EJ, Oldoni TL, Alencar SM, Reis A, Loguercio AD, Grande RH. Antioxidant activity by DPPH assay of potential solutions to be applied on bleached teeth. Braz Dent J. 2012;23(1):22-7. https://doi.org/10.1590/S0103-64402012000100004

12. Herrera DR, Durand-Ramirez JE, Falcão A, Silva EJ, Santos EB, Gomes BP. Antimicrobial activity and substantivity of Uncaria tomentosa in infected root canal dentin. Braz Oral Res. 2016;30(1):e61. https://doi.org/10.1590/1807-3107BOR-2016.vol30.0061

13. Siqueira Junior JF, Rôças ID, Marceliano-Alves MF, Pérez AR, Ricucci D. Unprepared root canal surface areas: causes, clinical implications, and therapeutic strategies. Braz Oral Res. 2018 Oct;32 suppl 1:e65. https://doi.org/10.1590/1807-3107bor-2018.vol32.0065

14. Brezhnev A, Neelakantan P, Tanaka R, Brezhnev S, Fokas G, Matinlinna JP. Antibacterial additives in epoxy resin-based root canal sealers: a focused review. Dent J (Basel). 2019 Jul;7(3):E72. https://doi.org/10.3390/dj7030072 
15. Silva EJ, Rosa TP, Herrera DR, Jacinto RC, Gomes BP, Zaia AA. Evaluation of cytotoxicity and physicochemical properties of calcium silicate-based endodontic sealer MTA Fillapex. J Endod. 2013 Feb;39(2):274-7. https://doi.org/10.1016/i.joen.2012.06.030

16. Tuğ Kılkış B, Er K, Taşdemir T, Yildirim M, Taskesen F, Tümkaya L, et al. Neurotoxicity of various root canal sealers on rat sciatic nerve: an electrophysiologic and histopathologic study. Clin Oral Investig. 2015 Nov;19(8):2091-100. https://doi.org/10.1007/s00784-015-1447-y

17. Gandolfi MG, Siboni F, Prati C. Chemical-physical properties of TheraCal, a novel light-curable MTA-like material for pulp capping. Int Endod J. 2012 Jun;45(6):571-9. https://doi.org/10.1111/j.1365-2591.2012.02013.x

18. Zhang $H$, Shen Y, Ruse ND, Haapasalo M. Antibacterial activity of endodontic sealers by modified direct contact test against Enterococcus faecalis. J Endod. 2009 Jul;35(7):1051-5. https://doi.org/10.1016/i.joen.2009.04.022

19. Yoshino P, Nishiyama CK, Modena KC, Santos CF, Sipert CR. In vitro cytotoxicity of white MTA, MTA Fillapex® and Portland cement on human periodontal ligament fibroblasts. Braz Dent J. 2013;24(2):111-6. https://doi.org/10.1590/0103-6440201302115

20. Silva EJ, Accorsi-Mendonça T, Pedrosa AC, Granjeiro JM, Zaia AA. Long-Term Cytotoxicity, pH and dissolution rate of AH Plus and MTA Fillapex. Braz Dent J. 2016 Jul-Aug;27(4):419-23. https://doi.org/10.1590/0103-6440201600735

21. Prati C, Gandolfi MG. Calcium silicate bioactive cements: biological perspectives and clinical applications. Dent Mater. 2015 Apr;31(4):351-70. https://doi.org/10.1016/i.dental.2015.01.004 